\title{
Société Suisse de Cardiologie. Schweizerische
}

\section{Kardiologische Gesellschaft}

Séance du 9 mai 1958 à Lausanne. Sitzung am 9. Mai 1958 in Lausanne

Verzeichnis der Vorträge — Liste des Communications

Ferrero, C. et Gay, E. (Geneve): Modification de $\Gamma$ onde U de

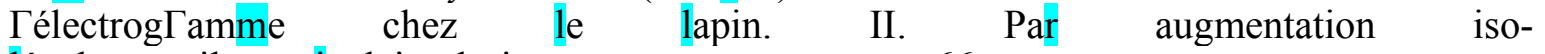
lée du travail ventriculaire droit 66

Effert, S., Domaníg, E. und Erkens, H. (Düsseldorf): Möglichkeiten des Ultraschall-Echoverfahrens in der Herzdiagnostik

Scholer, H. und Sokhegyí, D. (Liestal): Flüchtige polymorphe EKG-Veränderungen bei Hitzschlägen ... 81

Gürtler, R., Weber, J. W., Mailer, P., Sahli, H. R. und Cottier, H. (Bern): Besonderheiten beim Ebstein-Syndrom 90

Rìier, J. L., Reymond, $\quad$ C. L., Desbaillets, $P . \quad$ (Lausanne) et Duchosal, $\quad P . \quad W$. (Geneve): Le diagnostic de la commu nication interauriculaire basse. .95

Mailer, P. (Bern): Zur Klinik und Therapie der Vorhof-

septumdefekte und Pulmonalstenosen $\quad \ldots 110$

Pauli, H. G. (Bern), Noe F.E. und Coates, E. O. (Detroit, Mich.): Die zentrale Atemregulation bei Herzpatienten 114

Imhof, P., Hürlimann, A. und Steinmann, B. (Bern): Periphere Kreislaufuntersuchungen mil synthetischem Hypertensin II ..... 120

Kipfer, K. (Bern): Postural Hypotension; ein Therapieversuch mit synthetischem Hypertensin II . . . 131

Strausak, A., Cottier, P. und Schmid, A. (Bern): Aethyl-7oxyaceto-flavon (Recordil) in der Behandlung der An gina pectoris und seine Wirkung auf Hämodynamik und Nierenfunktion 138 